\title{
Power Transformers Differential Protection Using the p-q Power Theory
}

\author{
Luís M.R. Oliveira ${ }^{1,3}$ and Antonio J. Marques Cardoso ${ }^{2,3}$ \\ ${ }^{1}$ Instituto Superior de Engenharia, Universidade do Algarve, Portugal \\ ${ }^{2}$ University of Beira Interior, Department of Electromechanical Engineering, Portugal \\ ${ }^{3}$ Instituto de Telecomunicações, Department of Electrical and Computer Engineering, \\ University of Coimbra, Pole II, P - 3030-290 Coimbra, Portugal \\ lolivei@ualg.pt, ajmcardoso@ieee.org
}

\begin{abstract}
This paper describes the application of the p-q power theory to the differential protection of power transformers. The information provided by the harmonic content of the differential active and reactive power components is used to detect winding insulation failures and to distinguish them from magnetizing inrush current transients. A variety of test cases is presented in the paper, demonstrating the effectiveness of the protection strategy.
\end{abstract}

Keywords: Transformers, differential protection, p-q power theory.

\section{Introduction}

The vast majority of transformer differential relaying schemes use the second harmonic restraint method to discriminate between energization transients and internal faults. Harmonic restraint is based on the fact that the inrush current typically contains a large second-harmonic component, which is used to inhibit the relay operation during the transformer energization. However, inrush current parameters (peak, $2^{\text {nd }}$ harmonic and duration) of today's power transformers differ from those of older designs due to the use of higher grain oriented core steels, the step-lap core joint type, and higher rated design core induction values [1]. In modern power transformers, the $2^{\text {nd }}$ harmonic during inrush may be very low, jeopardizing relay security [2]. Moreover, the $2^{\text {nd }}$ harmonic component may also be generated during internal faults in the transformer, with relevant magnitudes.

Researchers and designers have made remarkable progress in the development of protection strategies since microprocessor-based relays were adopted for the differential protection of power transformers. A wide variety of protection schemes have been proposed, many of them combining different methods, such as the improvement of the classical harmonic restraint schemes [2], [3], the introduction of new trip and blocking signals [2], [4-7], the use of wave-shape recognition methods [3], and the application of advanced digital signal processing tools, fuzzy logic and artificial intelligence techniques [8]. 
Many of these strategies have proved to be successful to some extent, but very few have been incorporated in commercially available relays, where the $2^{\text {nd }}$ harmonic restraint method is still widely used, regardless of its limitations.

This paper describes the application of the p-q power theory for the discrimination between inrush currents and internal faults in transformers differential protection. The so-called Power Signature Analysis, which has been previously used to diagnose AC motors faults [9], is applied to the difference between the instantaneous powers at all the transformer's terminals. The information provided by the harmonic content of the resultant differential $\mathrm{p}-\mathrm{q}$ power signals is used for the discrimination between inrush transients and interturn short-circuits. With this approach, the traditional $2^{\text {nd }}$ harmonic restraint limitations are avoided.

\section{Contribution to Value Creation}

In today's digital era, an affordable and reliable electricity supply is essential to the economic and social progress. Simultaneously, the development of deregulation in power systems leads to a higher requirement on power quality. In the area of relay protection this means that a faster and sensitive protection is needed, while undesirable operation of the protection system is almost unacceptable [10]. It is therefore of crucial importance the development of new transformer protection schemes, which can improve the sensitivity of differential relays to detect internal faults and which can also provide a solution to the second harmonic restraint drawbacks. This will be of great value to improve the overall grid reliability, which is the key to the social and industrial development.

\section{Simulated Test System: Power and Current Transformer Models}

A three-phase, two winding, three leg, $10.3 \mathrm{kVA}, 230 / 132 \mathrm{~V}$ transformer model was previously developed for winding faults studies and it was used for the evaluation of the proposed protection strategy. The non-linear model is based on the combination of both magnetic and electric lumped-parameters equivalents circuits, which allows the modeling and simulation of the transformer in its natural electromagnetic environment. The faults are introduced in the model by dividing the affected winding into two parts, which represent the healthy and the faulty subwindings, Fig. 1(a). The current in the shorted turns is adjusted by varying the value of the fault impedance. A detailed description of the model implementation and/or validation, under inrush and fault conditions, can be found in [6], [11], [12].

The influence of the current transformers (CT) core saturation on the functionality of the protective system is also considered in the simulation study: the current inputs of the differential relay are computed by using a non-linear CT model. The CT model is based on the same principles used to simulate the behavior of the power transformer. The diagram of the simulated test system is shown in Fig. 1(b). 
(a)

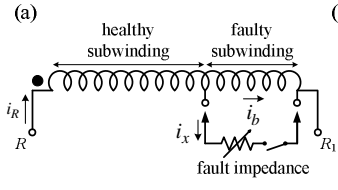

(b)

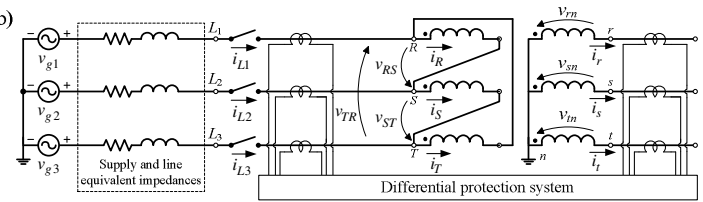

Fig. 1. (a) Equivalent circuit for a winding fault; (b) diagram of the test system

\section{Protection Strategy Based on the p-q Power Theory}

The active power differential method was firstly proposed in [7] for the discrimination between inrush transients and internal faults in power transformers. The differential power is computed as the difference between the instantaneous powers at all the transformer's terminals. In [7], the average of the instantaneous differential power is responsible for both the operation and the blocking signals of the relay. The discrimination criterion is based on the following basic principle: the average power is almost zero for energizing, but an internal fault consumes large power. However, the direct application of the average power as a decision signal for the discrimination of inrush/fault conditions can lead to misleading results, due to the oscillations of the average active power, as reported in [7].

The same basic principle mentioned before is particularly suitable for the application of the p-q theory to the power differential method, where the active power component is directly related to the internal fault within the transformer and the reactive power component is associated with the inrush transient.

\subsection{Differential Power Components Computation}

A two-winding transformer with a $D Y n 5$ winding connection [Fig. 1(b)] is considered here, but the method is valid for any multi-winding transformer. For simplicity, the differential power components are computed using phase quantities. The d-q components of the voltage and current, of high and low voltage sides of the transformer, are computed by applying the Park's transformation:

$$
\begin{gathered}
{\left[\begin{array}{ll}
v_{D 1} & v_{Q 1}
\end{array}\right]^{T}=T \cdot\left[\begin{array}{lll}
v_{R S} & v_{S T} & v_{T R}
\end{array}\right]^{T},} \\
{\left[\begin{array}{ll}
v_{D 2} & v_{Q 2}
\end{array}\right]^{T}=T \cdot\left[\begin{array}{lll}
v_{r n} & v_{s n} & v_{t n}
\end{array}\right]^{T},} \\
{\left[\begin{array}{ll}
i_{D 1} & i_{Q 1}
\end{array}\right]^{T}=T \cdot\left[\begin{array}{lll}
i_{R} & i_{S} & i_{T}
\end{array}\right]^{T},} \\
{\left[\begin{array}{ll}
i_{D 2} & i_{Q 2}
\end{array}\right]^{T}=T \cdot\left[\begin{array}{lll}
i_{r} & i_{s} & i_{t}
\end{array}\right]^{T},}
\end{gathered}
$$

where $T$ is the Park's transformation matrix:

$$
T=\left[\begin{array}{ccc}
\sqrt{2} / \sqrt{3} & -1 / \sqrt{6} & -1 / \sqrt{6} \\
0 & 1 / \sqrt{2} & -1 / \sqrt{2}
\end{array}\right] .
$$


Then, the primary- and secondary-side Park's power components $(k=1$ and $k=2$, respectively) are computed:

$$
\begin{aligned}
& p_{k}=v_{D k} \cdot i_{D k}+v_{Q k} \cdot i_{Q k}, k=1,2, \\
& q_{k}=v_{Q k} \cdot i_{D k}-v_{D k} \cdot i_{Q k}, k=1,2,
\end{aligned}
$$

and finally the differential active and reactive power components are:

$$
\begin{aligned}
& p_{d(\text { load dependent })}=p_{1}-p_{2}, \\
& q_{d(\text { load dependent })}=q_{1}-q_{2} .
\end{aligned}
$$

The sensitivity of the method can be further enhanced by compensating for the internal active and reactive load-dependent power components. The winding copper losses can be subtracted in (8):

$$
p_{d}=p_{1}-p_{2}-R_{1} \cdot\left(i_{D 1}^{2}+i_{Q 1}^{2}\right)-R_{2} \cdot\left(i_{D 2}^{2}+i_{Q 2}^{2}\right),
$$

where $R_{1}$ and $R_{2}$ are the primary- and secondary-side winding resistances, respectively. Similarly, the reactive power supplied to the short-circuit reactance can be deducted in (9):

$$
q_{d}=q_{1}-q_{2}-L_{1} \cdot\left(d i_{Q 1} / d t \cdot i_{D 1}-d i_{D 1} / d t \cdot i_{Q 1}\right)-L_{2} \cdot\left(d i_{Q 2} / d t \cdot i_{D 2}-d i_{D 2} / d t \cdot i_{Q 2}\right),
$$

where $L_{1}$ and $L_{2}$ are the primary- and secondary-side leakage inductances, respectively.

\subsection{Differential Power Signature During an Internal Fault}

Fig. 2 presents the results for the case of a winding fault with 6 shorted turns in the primary winding of phase $R$. The current in the fault impedance is limited to 5 times the rated current of the affected winding. The transformer operates at full load, with 0.8 power factor.

With these conditions the fundamental component of the differential current associated with the faulty phase increases about 7 times when the short-circuit occurs. This differential signal reaches up to $20 \%$ of the transformer winding rated current, which is in the range of the minimum differential protection pickup (e.g. 15\%-20\%) typically required for the operation of the relay. Therefore, it can be assumed that this fault would be the smallest interturn insulation defect that can be detected by the traditional protection systems. It should be noted, however, that a higher value of the minimum protection pickup setting is often used, in order to deal with the ratio and phase mismatches and avoid false trips of the protection system. In this case the turnto-turn fault may not be detectable initially and the winding protection is uncertain.

Fig. 2(a) presents the evolution of the instantaneous differential active and reactive powers during the turn-to-turn winding fault occurrence. After the fault inception there is an increase in the magnitude of both instantaneous power components. They have an oscillatory component at twice the supply frequency $\left(f_{\text {supply }}=50 \mathrm{~Hz}\right)$. 
A further insight into the characteristics of the differential power components can be obtained by performing the frequency analysis of the power signals. Fig. 2(b) presents the relevant harmonic content of the differential active power during the fault transient. There is an increase in the DC and the $100 \mathrm{~Hz}$ components of $p_{d}\left(p_{d(D C)}\right.$ and the $p_{d(100)}$, respectively) as a consequence of the fault occurrence. After the short transient at the time of the fault introduction, the $50 \mathrm{~Hz}$ component, $p_{d(50)}$, is negligible. It seems that the $p_{d(D C)}$ and the $p_{d(100)}$ components can be used for fault detection. However, as explained in the next subsection, it is not possible to discriminate between internal faults and inrush transients by using the $100 \mathrm{~Hz}$ component of the differential active power.

The evolution of the relevant harmonic components of the differential reactive power is presented in Fig. 2(c). Only the $100 \mathrm{~Hz}$ component is affected by the fault occurrence, but some limitations also exist in using this parameter as a fault/inrush discriminator. This will be addressed later.

The DC value of the active power is substantially affected by the presence of the fault. The average value of the active power increases about 8 times as a result of the winding fault occurrence, which results in only a slightly improvement of the fault detection sensitivity, as compared to the fundamental component of the line currents involved with the affected phase (which increases 7 times for the same type of fault, as mentioned before). However, the differential power method is not affected by the ratio mismatch due to on-load tap-changer, and enhanced fault detection sensitivity is thus achieved. Therefore, the value of $p_{d(D C)}$ is a reliable indicator of the presence of a fault, and can be used as a trip signal of the relay.

To detect this low-level turn-to-turn fault the trip signal should be activated when $p_{d(D C)}$ exceeds a threshold of $800 \mathrm{~W}$. This is about 8 times the normal operating value (which is equal to the no-load losses). This allows the power differential relay to accommodate the measurement errors without nuisance tripping and still providing reliable fault detection.

\subsection{Differential Power Signature during an Inrush Transient}

Fig. 3(a) presents the waveforms of the instantaneous active and reactive powers during a transformer energization transient. It is considered in this case that the CT's reproduces correctly the inrush currents. The reactive component is largely affected during the energization of the transformer, in order to supply the magnetic energy to be stored in the windings. Comparatively, the instantaneous active power is much smaller. Moreover, its DC value $\left(p_{d(D C)}\right)$ has a reduced variation and never reaches the trip threshold value (800 W), as seen in Fig. 3(b).

The instantaneous reactive power has an asymmetric waveform, with a frequency of $50 \mathrm{~Hz}$ (and not $100 \mathrm{~Hz}$, as in the internal fault case). Accordingly, the $q_{d(\mathrm{DC})}$ and the $q_{d(50)}$ components have high values during the inrush transient, Fig. 3(c).

Figs. 3(d) to 3(f) present the same curves as in Figs. 3(a) to 3(c), but now considering the current transformers under saturated conditions. The DC component of the inrush currents drives the CT's into saturation, resulting in distorted secondary-side CT's current waveforms, with minor peak values, faster decay and slightly more symmetrical and bipolar waveforms, as compared to the non-saturated CTs case [6]. This results in smaller values of $q_{d}$ and $p_{d}$, Fig. 3(d). The influence of the CT saturation on the harmonic content of the $\mathrm{p}-\mathrm{q}$ power components during the inrush transient can be summarized as follows: 

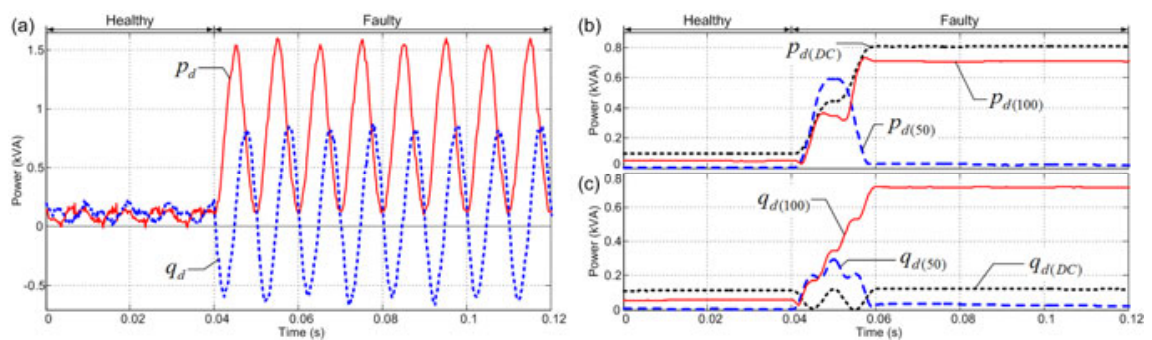

Fig. 2. Internal fault occurrence: (a) instantaneous waveforms of $p_{d}$ and $q_{d}$. DC, $50 \mathrm{~Hz}$ and 100 $\mathrm{Hz}$ harmonic components of: (c) $p_{d}$ and (d) $q_{d}$.
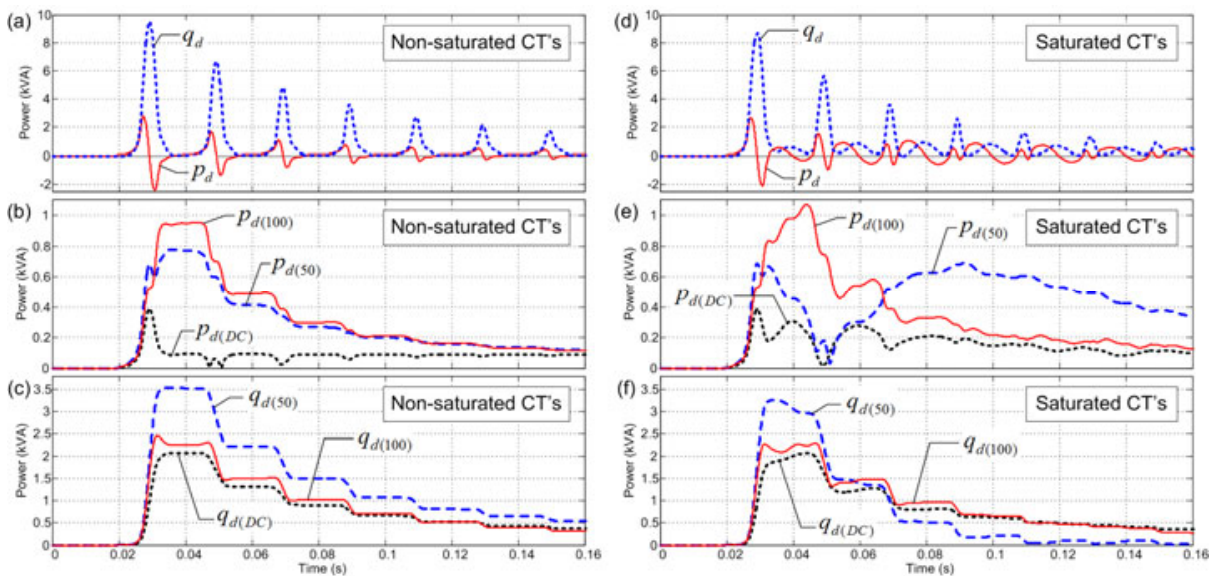

Fig. 3. Transformer energization transient. (a), (d): Instantaneous waveforms of $p_{d}$ and $q_{d}$. (b), (e): Harmonic components of $p_{d}$. (c), (f): Harmonic components of $q_{d}$. Transformer operation under no-load conditions. Energization at $t=20 \mathrm{~ms}$.

- The $p_{d(D C)}$ take larger values, but never reaches the trip threshold, Fig. 3(e).

- The behaviour of the $p_{d(50)}$ is irregular and very dependent upon the CT's saturation conditions.

- The $q_{d(\mathrm{DC})}$ and the $q_{d(50)}$ components have smaller values than in the nonsaturated CT's case, Fig. 3(f). Even so, at the inrush start these values are at least 20 times higher than in the case of an internal fault, Fig. 2(c).

The DC value and the $50 \mathrm{~Hz}$ harmonic component of the reactive power takes high values at the beginning of the energization transient, falling then slowly, with the same rate as the inrush currents, to the steady-state value. These two components are not affected by the interturn fault conditions, as seen before, and can be used to block the relay operation and avoid false trips.

The $p_{d(100)}$ and the $q_{d(100)}$ harmonic components are largely affected by both fault and inrush conditions, not allowing to discriminate between these two situations. 


\subsection{Inrush Blocking Signal}

The transformer energization can be detected by using the ratio between the signals that characterize the inrush transient $\left(q_{d(\mathrm{DC})}\right.$ and $\left.q_{d(50)}\right)$ and the trip indicator $\left(p_{d(\mathrm{DC})}\right)$. Accordingly, the proposed blocking signal is defined as:

$$
\text { blocking signal }=\left(\left|q_{d(D C)}\right|+\left|q_{d(50)}\right|\right) /\left|p_{d(D C)}\right| .
$$

The relay operation will be inhibited if the blocking signal is above a predefined threshold value. For the present case this threshold value can be assumed to be 10 . A safety margin is introduced between the threshold and the normal operation status, to avoid blocking the relay trip signal under the simultaneous occurrence of inrush currents and internal faults. It is considered here that the threshold is about 8 times the normal operating value.

In the case of an inrush event, although the trip indicator never reaches the $800 \mathrm{~W}$ threshold, the blocking signal increases promptly and the relay is inhibited during the first cycles of the transient, as seen in Fig. 4(a). When a fault occurs the blocking signal has the opposite behavior, i.e., decreases to a value which is 10 times smaller than the one obtained under normal operating conditions, Fig. 4(b).

Fig. 5 presents the evolution of the trip indicator and the blocking signal for the case of a simultaneous occurrence of a transformer energization and a winding turn-to-turn fault (with the same severity as in subsection 4.2). It can be seen that the threshold value of the blocking signal is not reached and the relay, which is rapidly triggered by the large value of $p_{d(\mathrm{DC})}$, is not blocked.

Other simulation studies, carried out under different inrush and fault conditions, lead to similar conclusions to the ones presented before.

\section{Conclusions}

This work explores the possibility of applying the Park's power components to solve the inrush/fault discrimination dilemma in power transformers differential protection.

The results indicate that the proposed p-q power-based strategy provides reliable inrush stabilization and enhanced sensitivity for internal faults.
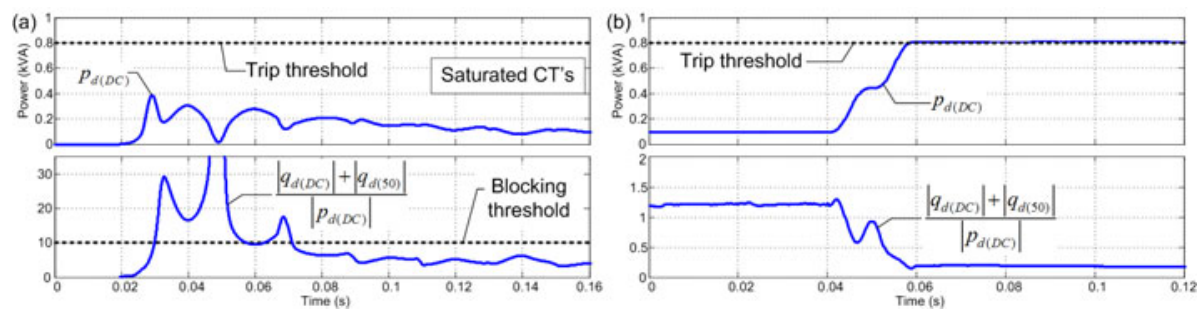

Fig. 4. Trip indicator and blocking signal, during: (a) energization (same conditions as in Fig. 3); (b) interturn fault occurrence (same conditions as in Fig. 2) 


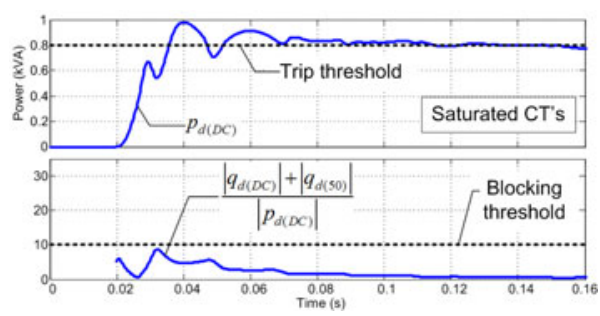

Fig. 5. Trip indicator and blocking signal, during a faulted transformer energization transient

With this approach, the traditional $2^{\text {nd }}$ harmonic restraint shortcomings are avoided. Additionally, the error introduced by the current mismatch due to the tap-changer is minimized, and increased sensitivity is obtained in the differential protection system.

\section{References}

1. Girgis, R.S., teNyenhuis, E.G.: Characteristics of Inrush Current of Present Designs of Power Transformers. In: Power Engineering Society General Meeting (2007)

2. Kasztenny, B., Kulidjian, A.: An Improved Transformer Inrush Restraint Algorithm Increases Security While Maintaining Fault Response Performance. In: Proc. 53rd Annual Conf. for Protective Relay Engineers (2000)

3. Guzmán, A., Zocholl, S., Benmouyal, G., Altuve, H.J.: A Current-Based Solution for Transformer Differential Protection - Part I: Problem Statement. IEEE Trans. Power Delivery 16, 485-491 (2001)

4. Gajić, Z., Brnčić, I., Hillström, B., Ivankovic, I.: Sensitive Turn-to-Turn Fault Protection for Power Transformers. In: CIGRÉ Study Committee B5 Colloquium (2005)

5. Baoming, G., Almeida, A.T., Qionglin, Z., Xiangheng, W.: An Equivalent Instantaneous Inductance-Based Technique for Discrimination Between Inrush Current and Internal Faults in Power Transformers. IEEE Trans. Power Delivery 20, 2473-2482 (2005)

6. Oliveira, L.M.R., Cardoso, A.J.M.: An EPVA-Based Strategy for Power Transformers Differential Protection. In: Proc. Advanced Research Workshop on Modern Transformers (2010)

7. Yabe, K.: Power Differential Method for Discrimination Between Fault and Magnetizing Inrush Current in Transformers. IEEE Trans. Power Delivery 12, 1109-1118 (1997)

8. Tripathy, M., Maheshwari, R.P., Verma, H.K.: Advances in Transformer Protection: a Review. Electric Power Components and Systems 33, 1203-1209 (2005)

9. Drif, M., Cardoso, A.J.M.: The Use of the Instantaneous-Reactive-Power Signature Analysis for Rotor-Cage-Fault Diagnostics in Three-Phase Induction Motors. IEEE Trans. Industrial Electronics 56, 4606-4614 (2009)

10. Lotfi-fard, S., Faiz, J., Iravani, R.: Improved Overcurrent Protection Using Symmetrical Components. IEEE Trans. Power Delivery 22, 843-850 (2007)

11. Oliveira, L.M.R., Cardoso, A.J.M.: A Permeance-Based Transformer Model and its Application to Winding Interturn Arcing Fault Studies. IEEE Trans. Power Delivery 25, 1589-1598 (2010)

12. Oliveira, L.M.R., Cardoso, A.J.M.: Power Transformers Behavior Under the Occurrence of Inrush Currents and Turn-To-Turn Winding Insulation Faults. In: Proc. Int. Conf. Electrical Machines (2010) 